nehmend an Bedeutung gewinnt. Sie sind eine beginnende zivilgesellschaftliche Bewegung, die bisher nicht im öffentlich-rechtlichen Sinne verankert sind. Ihr derzeitiger Schwerpunkt liegt auf der städtischen/kommunalen Ebene, während die Länder- und Bundesebene, vor allem auch wegen des Graswurzel-Charakters vieler Ernährungsräte, bisher kaum eine Rolle spielen. Allerdings verbreitet sich derzeit die Idee, Ernährungsräte auch auf höheren politisch-administrativen Ebenen einzusetzen. Im kürzlich veröffentlichten Bericht des International Panel of Experts on Sustainable Food Systems (iPES Food) wird die Unterstützung der Etablierung eines Ernährungsrats auf EU-Ebene als eine politische Maßnahme im Rahmen einer neuen Governance-Architektur für nachhaltige Ernährungssysteme vorgeschlagen. ${ }^{53}$

Möglicherweise kann eine stärkere rechtliche Einbindung von Ernährungsräten zu einer besseren Durchsetzung der Belange im Hinblick auf eine Ernährungswende führen und z.B. zur Verringerung von Lebensmittelabfällen beitragen. Auf der anderen Seite sollte eine solche Bewegung von unten nicht durch zu viele formale Anforderungen abgewürgt werden. Wünschenswert wäre, wenn öffentliche Entscheidungsträger zumindest Bereitschaft zeigen würden, die Belange von Ernährungsräten anzuhören und das zivilgesellschaftliche Engagement im Rahmen ihrer Möglichkeiten zu unterstützen. Dies gilt z.B. für die Förderung von Projekten, ggf. durch Abordnung von Mitarbeitern aus der Verwaltung zur Mitarbeit in den Räten, für die Bereitstellung von Räumlichkeiten und Koordinationsstellen etc. Ein wichtiges Signal kann weiter darin bestehen, wenn Kommunen dem Mailänder Abkommen über städtische Ernährungspolitik (Milan Urban Food Policy Pact) vom 15.10.2015 als politischem Bezugspunkt für proaktives Handeln beitreten. Hierin anerkennen die Mitgliedsstädte unter anderem deren zentrale Rolle bei der Entwicklung nachhaltiger Ernährungssysteme, und sie erklären, dass sie ihre kommunale Ernährungspolitik in ihre sozialen, ökonomischen und Umweltpolitiken sowie -programme integrieren. ${ }^{54}$ Bisher haben 197 Städte den Pakt unterzeichnet, darunter allerdings nur drei deutsche: Frankfurt, Köln und Berlin (2015). Ernährungsräte können dazu beitragen, ihre jeweiligen Kommunen zur Teilnahme an diesem Pakt zu bewegen und im Hinblick auf eine Ernährungswende aktiv zu werden.

Insgesamt bieten Ernährungsräte die Chance, in der gesamten Kette, von der Erzeugung über die Verarbeitung und den Transport bis zum Verbrauch und zur Vermeidung und Entsorgung von Lebensmittelabfällen, eine Wende in Richtung auf eine nachhaltigere Ernährung zu fördern. Dazu bedarf es zivilgesellschaftlichen Engagements und der Aufgeschlossenheit und Bereitschaft privater und öffentlicher Stellen, die Bildung und Arbeit von Ernährungsräten zu unterstützen - nicht zuletzt auch durch die Schaffung geeigneter rechtlicher Rahmenbedingungen.

Open Access. Dieser Artikel wird unter der Creative Commons Namensnennung 4.0 International Lizenz veröffentlicht, welche die Nutzung, Vervielfältigung, Bearbeitung, Verbreitung und Wiedergabe in jeglichem Medium und Format erlaubt, sofern Sie den/die ursprünglichen Autor(en) und die Quelle ordnungsgemäß nennen, einen Link zur Creative Commons Lizenz beifügen und angeben, ob Änderungen vorgenommen wurden.

Die in diesem Artikel enthaltenen Bilder und sonstiges Drittmaterial unterliegen ebenfalls der genannten Creative Commons Lizenz, sofern sich aus der Abbildungslegende nichts anderes ergibt. Sofern das betreffende Material nicht unter der genannten Creative Commons Lizenz steht und die betreffende Handlung nicht nach gesetzlichen Vorschriften erlaubt ist, ist für die oben aufgeführten Weiterverwendungen des Materials die Einwilligung des jeweiligen Rechteinhabers einzuholen.

Weitere Details zur Lizenz entnehmen Sie bitte der Lizenzinformation auf http://creativecommons.org/licenses/by/4.0/deed.de.

Open Access funding enabled and organized by Projekt DEAL.

53) De Schutter/Jacobs/Clément/Ajena, Towards a Common Food Policy for the European Union, 2019, Stand 16.8.2020, abrufbar unter http://www.ipes-food.org/pages/CommonFoodPolicy, S. 36-37.

54) Milan Urban Food Policy Pact, Stand 5.7.2019, abrufbar unter http://www.milanurbanfoodpolicypact.org/signatory-cities/.

\title{
https://doi.org/10.1007/s10357-020-3749-3
}

\section{Europäisierung des Individualrechtsschutzes im Umweltrecht}

\author{
Anmerkung zum Urteil des Europäischen Gerichtshofs v. 3.10.2019 - C-197/18 \\ Dominik Römling
}

(C) Der/die Autor(en) 2020. Dieser Artikel ist eine Open-Access-Publikation.

Während die Fortentwicklung des Verbandsklagerechts nach Art. 9 Abs. 3 der Aarhus-Konvention mit der Protect-Entscheidung des Europäischen Gerichtshofs ihren vorläufigen Schlusspunkt erreicht hat, besteht die Diskussion um die Klagerechte Einzelner aus der

Dominik Römling, Wissenschaftlicher Mitarbeiter

am Institut für Umwelt- und Planungsrecht,

Westfälische Wilhelms-Universität,

Münster, Deutschland
Konvention fort. Sie erfährt jüngst durch das Urteil des EuGH in der Rs. C-197/18 neue Impulse. Der Beitrag analysiert die Reichweite der dort entwickelten Maßstäbe und legt die Konsequenzen für das Verständnis des Individualrechtsschutzes im deutschen Umweltrecht dar.

\section{Einführung}

Art. 9 Abs. 3 der Aarhus-Konvention (AK) hat die Klagerechte von Verbänden in erheblichem Maße erwei- 
tert. ${ }^{1}$ Den vorläufigen Schlusspunkt bildet die Entscheidung des Europäischen Gerichtshofs (EuGH) in der Rs. Protect, in der er Verbandsklagerechte auch jenseits der Fälle, in denen das umweltrechtliche Unionssekundärrecht eine Klagebefugnis vorsieht, für völkerrechtlich gefordert hält. ${ }^{2}$ Die Bedeutung dieser Entscheidung für die Rechtsstellung von Verbänden ist in der Literatur umfangreich aufgearbeitet worden. ${ }^{3}$ Weniger beachtet ist die Frage, inwieweit Art. 9 Abs. 3 AK in der Interpretation des EuGH Konsequenzen für die Klagerechte Einzelner nach sich zieht. ${ }^{4}$ Sie ist jedoch schon deshalb von Relevanz, weil der EuGH in der Entscheidung Protect Art. 9 Abs. 3 AK in Verbindung mit dem primär individualschützenden Rechtsschutzgrundrecht aus Art. 47 der Charta der Grundrechte der Europäischen Union (GRCh) liest, ${ }^{5}$ und gewinnt besondere Aktualität aus einem jüngeren Auslegungsvorschlag des EuGH. Angesprochen ist damit das Urteil in der Rs. Wasserleitungsverband Nördliches Burgenland. ${ }^{6}$ Im Folgenden werden Kontext und rechtliche Tragweite des Urteils vorgestellt und ihre Auswirkungen auf den Individualrechtsschutz im deutschen Umweltrecht erörtert. Anlass für den Abgleich mit den Anforderungen des deutschen Rechts bietet insbesondere, dass das BVerwG unter ausdrücklicher Rezeption des EuGH-Entscheidung in der Rs. C-197/18 eine Individualklagebefugnis aus $\$ 47 \mathrm{~d}$ BImSchG in Bezug auf die Fortschreibung eines Lärmaktionsplans abgelehnt hat.

\section{Das Urteil des EuGH in der Rs. C-197/18}

\subsection{Sachverhalt und Vorlagefrage}

Ausgangspunkt der Entscheidung in der Rs. Wasserleitungsverband Nördliches Burgenland sind die Anträge dreier Individualkläger auf Änderung oder Fortschreibung eines wasserrechtlichen Aktionsprogrammes, das durch das österreichische Bundesministerium für Nachhaltigkeit und Tourismus in Umsetzung von Art. 5 der Nitratrichtlinie $\left(\right.$ Nitrat-RL) ${ }^{8}$ erlassen wurde. Die Kläger standen in unterschiedlicher Weise mit dem Wasserrecht in Berührung: Es handelte sich zunächst um einen Wasserversorger in Gestalt einer Körperschaft des öffentlichen Rechts, ferner um eine Privatperson, die einen Hausbrunnen besitzt, sowie um einen Gemeinde, die einen kommunalen Brunnen betreibt. ${ }^{9}$ Ungeachtet der verschiedenen Organisationsformen der Kläger sind sie sämtlich als Individual- und nicht als Verbandskläger zu qualifizieren. Alle drei rügten den Nitratgehalt des von ihnen genutzten oder bereitgestellten Wassers.

Die Anträge der Kläger wurden vom Ministerium mit Verweis auf die fehlende Verfahrens- und Klagebefugnis zurückgewiesen: Nach österreichischem Recht setzt die Klagebefugnis natürlicher und juristischer Personen voraus, dass sie Träger subjektiver materieller Rechte sind, deren Verletzung sie geltend machen. ${ }^{10}$ Dies weist deutliche Nähe zum deutschen Verständnis der Klagebefugnis i.S.d. \42 Abs. 2 2. Hs. VwGO auf. ${ }^{11}$ Im konkreten Fall fehlte es nach Auffassung des Ministeriums an der Klagebefugnis, da die Vorschriften des österreichischen Wasserrechts, die auf der Nitrat-RL beruhen, nicht individualschützend seien. ${ }^{12}$ Das mit der Beschwerde gegen die Ablehnung der Anträge befasste Verwaltungsgericht Wien legte dem EuGH die Frage vor, ob das Unionsrecht verlangt, dass sich Individualkläger auf die Pflicht zur Aufstellung von wasserrechtlichen Aktionsprogrammen berufen können. ${ }^{13}$ Der EuGH fokussiert die Vorlagefrage auf die Prüfung, ob Art. 288 AEUV und Art. 5 Abs. 4, Abs. 5 Nitrat-RL dahingehend auszulegen sind, dass natürliche und juristische Personen, die mit der Wasserversorgung betraut sind oder einen Brunnen nutzen, die Fortschreibung von Aktionsprogrammen i.S.d. Art. 5 Abs. 5 Nitrat-RL verlangen können. ${ }^{14}$ Im Zentrum des Verfahrens steht damit die Frage, in welchem Umfang die volle Wirksamkeit des Unionsrechts verlangt, dass sich
Einzelne auf Bestimmungen der Wasserrahmenrichtlinie berufen können, inwieweit die Richtlinie also subjektive Rechte vermittelt.

\subsection{Entscheidung des EuGH}

Wie in seinen umweltprozessualen Urteilen üblich, ${ }^{15}$ leitet der EuGH die Beantwortung dieser Frage ein, indem er auf die Notwendigkeit der - auch gerichtlichen - Möglichkeit betroffener Personen, sich auf die Bestimmungen einer Richtlinie zu berufen, verweist. ${ }^{16}$ Konkret für das durch die Aarhus-Konvention überformte Unionsrecht bedeutet dies grundsätzlich einen aus Art. 9 Abs. 3 AK, Art. 47 GRCh abzuleitenden Anspruch auf gerichtlichen Schutz der durch das Unionsrecht verliehenen Rechte zugunsten von Mitgliedern der Öffentlichkeit, sofern sie im innerstaatlichen Recht festgelegte Kriterien erfüllen. ${ }^{17}$ Dieser Rechtsschutzanspruch erstreckt sich, so verstanden, sowohl auf Individualkläger als auch auf Umweltorganisationen. Die letztgenannten sind gem. Art. 2 Nr. 5 AK zwar insoweit privilegiert, als sie stets als betroffene Öffentlichkeit gelten, hierauf kommt aber es aus Sicht des EuGH für die grundsätzliche Notwendigkeit einer Rechtsschutzmöglichkeit nicht an: Weder Mitgliedern der Öffentlichkeit noch - und erst recht - Umweltorganisationen darf der Zugang

1) S. für einen jüngeren Überblick statt vieler Guckelberger, $\mathrm{NuR}$ 2020, 149, $150 \mathrm{ff}$.

2) EuGH, Urt. v. 20.12.2017 - C-664/15, NuR 2018, 395 - Protect, Rdnr. $30 \mathrm{ff}$.

3) Vgl. - jeweils mit unterschiedlichen Deutungen und Stoßrichtungen - Wegener, ZUR 2018, $217 \mathrm{ff}$.; Franzius, NVwZ 2018, 219 ff.; Sobotta, JEEPL 15 (2018), $241 \mathrm{ff}$; Schlacke/Römling, in: Schlacke/Schrader/Bunge (Hrsg.), Aarhus-Handbuch, 2. Aufl. 2019, §3 Rdnr. 57; Römling, ZEuS 2019, 147 ff.; Ruffert, DVB1. 2019, 1033 ff.; Durner, Rechtsgutachten zur Wiedereinführung der Präklusion, 2019, S. 18 ff.; Stöger, in: Kment (Hrsg.), Der Einfluss des Europäischen Gerichtshofs auf das Umwelt- und Infrastrukturrecht, 2020, S. $151 \mathrm{ff}$.

4) Franzius, NVwZ 2018, 219, 221 deutet insoweit an, dass eine schematische Unterscheidung von Individual- und Verbandsklage wohl nicht haltbar ist; ähnlich Wegener, ZUR 2018, 217, 221.

5) Näher zu dieser argumentativen Dimension des Urteils Römling, ZEuS 2019, 147, 158 ff.; krit. zum Rekurs des EuGH auf Art. 47 GRCh Fellenberg/Schiller, in: Landmann/Rohmer (Begr.), Umweltrecht, 89. EL 2019, Vorb. UmwRG Rdnr. 44; Ruffert, DVB1. 2019, 1033, $1036 \mathrm{ff}$.

6) EuGH, Urt. v. 3. 10.2019 - C-197/18, NuR 2019, 751 (Wasserleitungsverband Nördliches Burgenland).

7) BVerwG, Urt. v. 28.11.2019 - 7 C 2.18, juris.

8) Richtlinie 91/676/EWG des Rates vom 12.12.1991 zum Schutz der Gewässer vor Verunreinigung durch Nitrat aus landwirtschaftlichen Quellen, Abl. 1991 L 375, S. 1, zuletzt geändert durch die Verordnung (EG) Nr. 1137/2008 des Europäischen Parlaments und des Rates vom 22.10.2008, Abl. 2008 L 311, S. 1

9) Vgl. EuGH, Urt. v. 3. 10.2019 - C-197/18, NuR 2019, 751 (Wasserleitungsverband Nördliches Burgenland), Rdnr. 16ff.

10) Vgl. EuGH, Urt. v. 3. 10.2019 - C-197/18, NuR 2019, 751 (Wasserleitungsverband Nördliches Burgenland), Rdnr. 19.

11) Epiney, NVwZ 2014, 465, 467.

12) Vgl. EuGH, Urt. v. 3. 10.2019 - C-197/18, NuR 2019, 751 (Wasserleitungsverband Nördliches Burgenland), Rdnr. 19

13) Vgl. EuGH, Urt. v. 3.10.2019 - C-197/18, NuR 2019, 751 (Wasserleitungsverband Nördliches Burgenland), Rdnr. $20 \mathrm{ff}$.

14) Vgl. EuGH, Urt. v. 3. 10.2019 - C-197/18, NuR 2019, 751 (Wasserleitungsverband Nördliches Burgenland), Rdnr. 28.

15) EuGH, Urt. v. 8.11.2016 - C-243/15, ZUR 2017, 86 (Lesoochranárske zoskupenie VLK), Rdnr. 44; EuGH, Urt. v. 20.12. 2017 - C-664/15, NuR 2018, 395 (Protect), Rdnr. 34.

16) EuGH, Urt. v. 3. 10. 2019 - C-197/18, NuR 2019, 751 (Wasserleitungsverband Nördliches Burgenland), Rdnr. $30 \mathrm{f}$.

17) EuGH, Urt. v. 3. 10. 2019 - C-197/18, NuR 2019, 751 (Wasserleitungsverband Nördliches Burgenland), Rdnr. 33. 
zu Gericht am Maßstab des Unions- und Völkerrechts verwehrt werden. ${ }^{18}$

Der Rückgriff auf Art. 47 GRCh ist dabei nicht neu. Er liegt, da die Rechtsschutzgarantie aus Art. 47 GRCh als Garantie effektiven Individualrechtsschutzes zu verstehen ist, ${ }^{19}$ im Hinblick auf die hier in Rede stehenden Individualklagen jedoch näher als in den Urteilen, in denen er bisher aktiviert wurde und die stets Verbandsklagen zum Gegenstand hatten. ${ }^{20}$ Jedenfalls dürfte die Verknüpfung von Art. 9 Abs. 3 AK und Art. 47 GRCh nun als gefestigte Rechtsprechung gelten. Sie eröffnet, indem das von Art. 47 GRCh verlangte Schutzniveau gerade für die in Rede stehenden Rechte zu ermitteln ist, ${ }^{21}$ die Analyse der Zielsetzung und der maßgeblichen Bestimmungen der Nitrat-RL mit Blick auf ihre individualschützende Wirkung.

Der EuGH knüpft diese Analyse an den Terminus der Verunreinigung i.S.d. Art. 2 j) Nitrat-RL: Liegt eine solche Verunreinigung vor, ist die rechtmäßige Nutzung der Gewässer behindert. ${ }^{22}$ Schon hieraus folge die Einschränkung der Rechte nutzungsberechtigter Personen. ${ }^{23}$ Maßgeblich ist dann nicht mehr eine konkret-subjektive Gesundheitsgefährdung, ${ }^{24}$ sondern es genügt die objektive Verunreinigung des benutzten Gewässers, an dem ein subjektives Nutzungsrecht besteht. So wird der individualisierte Ansatz zur Ermittlung des Klagebefugnis im Grundsatz beibehalten, da das subjektive Nutzungsrecht der Bezugspunkt des Schutzes bleibt. Er wird aber - gerade im Vergleich zur im Ausgangspunkt ähnlichen Janecek-Entscheidung - erweitert, da schon die objektive Überschreitung eines normativen Grenzwerts zur Ermittlung der individualschützenden Wirkung beitragen kann. Demgegenüber hingen in der Entscheidung Janecek die unmittelbare Betroffenheit und, akzessorisch, die Klagebefugnis neben der objektiven Grenzwertüberschreitung noch von einer konkreten Gesundheitsgefährdung durch die objektive Rechtsverletzung ab. ${ }^{25}$ Auf dieses Kriterium kommt es für die unmittelbare Betroffenheit in der Lesart der Entscheidung Wasserleitungsverband Nördliches Burgenland nicht mehr an. Bei alldem bleibt indes von Bedeutung, dass die objektive Verunreinigung durch Kläger geltend gemacht wird, die ein eigenes Nutzungsrecht inne haben. Deutlich wird dies, wenn der EuGH ihre individuelle Situation in Bezug nimmt, um die Beeinträchtigung der Nutzungsmöglichkeit zu bestimmen. ${ }^{26}$ Ohne subjektives Nutzungsrecht am Wasser kämen eine Rechtsverletzung in Gestalt der Missachtung der Vorgaben aus der Nitrat-RL und damit eine Klagebefugnis nicht in Betracht.

\subsection{Keine Aufgabe der Unterscheidung zwischen Individual- und Verbandsklägern}

Der Befund, die Dichotomie von Verbands- u. Individualklage sei aufgegeben, ${ }^{27}$ kann deshalb nicht vollends überzeugen. Denn in der Protect-Entscheidung kam es für den EuGH nicht auf eine nach den vorstehenden - und zweifellos erweiterenden - Maßstäben zu ermittelnde individuelle Betroffenheit an. ${ }^{28}$ Ihre Prüfung war, da die klagende Umweltorganisation ohnehin gem. Art. 2 Nr. 5 AK privilegiert war, entbehrlich. ${ }^{29}$ So bleibt die strukturell-funktionale Unterscheidung ${ }^{30}$ zwischen Individual- und Verbandsklägern auch nach der Entscheidung Wasserleitungsverband Nördliches Burgenland gültig, wenngleich der Kreis der zur Individualklage Befugten neu vermessen wird. ${ }^{31}$ Die hier besprochene Entscheidung ist vor diesem Hintergrund keine Fortschreibung der Protect-Entscheidung, sondern wirkt komplementär, indem sie die Rechtsstellung der Individualkläger definiert.

\section{Bedeutung für Individualklagerechte} nach deutschem Recht

Unionsrechtliche Vorgaben für den Zugang Einzelner zu verwaltungsgerichtlichem Rechtsschutz sind im Wege ei- ner unionsrechtskonformen Auslegung des subjektiv-öffentlichen Rechts nach deutschem Verständnis und damit über $\$ 42$ Abs. 2 2. Hs. VwGO zu verarbeiten. ${ }^{32}$ Soweit also der Individualrechtsschutz auf Ebene des Unionsrechts erweitert wird, muss diese Erweiterung in Gestalt einer Klagebefugnis Einzelner vor den nationalen Gerichten gespiegelt werden.

Das BVerwG hatte bereits Gelegenheit, die Maßstäbe aus der Entscheidung in der Rs. C-197/18 anzuwenden. Zu beurteilen war die Klagebefugnis einer Individualklägerin in Bezug auf die Fortschreibung des Lärmaktionsplans für einen Verkehrsflughafen. ${ }^{33}$ Das BVerwG hielt die Klage zwar in ausdrücklicher Analogie zur Luftreinhalteplanung dem Begehren nach als allgemeine Leistungsklage für statthaft. ${ }^{34}$ Es qualifizierte die in einer Lärmsschutzzone wohnhafte Klägerin aber aus drei Gründen nicht für klagebefugt: Ers-

18) EuGH, Urt. v. 3. 10.2019 - C-197/18, NuR 2019, 751 (Wasserleitungsverband Nördliches Burgenland), Rdnr. 34.

19) Gärditz, Gutachten D zum 71. Deutschen Juristentag, 2016, S D21 ff.; eingehend zu diesem Verständnis d. Art. 47 GRCh Last, Garantie wirksamen Rechtsschutzes gegen Maßnahmen der Europäischen Union, 2008, S. 144 ff.; s. a. Hofmann, Rechtsschutz und Haftung im Europäischen Verwaltungsverbund, 2004, S. $189 f$.

20) Hieran entzündet sich im Wesentlichen die Kritik am Rückgriff auf Art. 47 GRCh, da die Norm ein vom Klagerecht unabhängiges Recht voraussetzt, vgl. Fellenberg/Schiller, in: Landmann/ Rohmer (Begr.), Umweltrecht, 89. EL 2019, Vorb. UmwRG Rdnr. 44; Gärditz, Die Verwaltung 52 (2019), 259, 294 f.; Erklärungsversuch bei Römling, ZEuS 2019, 147, $162 \mathrm{ff}$.

21) Rademacher, Realakte im Rechtsschutzsystem der Europäischen Union, 2014, S. 191; vgl. a. Bast, in: Leible/Terhechte (Hrsg.), Europäisches Rechtsschutz- und Verfahrensrecht, 2014, \$35 Rdnr. 7: „Art. 47 Abs. 1 GRCh definiert das materielle Schutzgut der gerichtlichen Rechtsschutzgarantie“.

22) EuGH, Urt. v. 3. 10.2019 - C-197/18, NuR 2019, 751 (Wasserleitungsverband Nördliches Burgenland), Rdnr. $37 \mathrm{ff}$.

23) EuGH, Urt. v. 3.10. 2019 - C-197/18, NuR 2019, 751 (Wasserleitungsverband Nördliches Burgenland), Rdnr. 40.

24) EuGH, Urt. v. 3. 10.2019 - C-197/18, NuR 2019, 751 (Wasserleitungsverband Nördliches Burgenland), Rdnr. 41.

25) EuGH, Urt. v. 25.7.2008 - C-237/07, Slg. 2008, I-6221 (Janecek), Rdnr. $35 \mathrm{ff}$

26) EuGH, Urt. v. 3. 10. 2019 - C-197/18, NuR 2019, 751 (Wasserleitungsverband Nördliches Burgenland), Rdnr. 42.

27) So Henn, ZUR 2019, 680, 680; weniger grundsätzlich Reinhardt, NVwZ 2019, 1591, 1592, der aber jedenfalls die Monopolstellung der Umweltverbände als nichtstaatliche Sachwalter der Effektuierung des europäischen Umweltrechts gefallen sieht; in diese Richtung auch Berkemann, DVBl. 2020 (i.E.)

28) Zutreffend Wegener, ZUR 2018, 217, 220.

29) Vgl. EuGH, Urt. v. 20.12.2017 - C-664/15, NuR 2018, 395 (Protect), Rdnr. 39.

30) Vol. Gärditz, Die Verwaltung 52 (2019), 259, 295

31) Zutreffend Köck/Henn, NVwZ 2020, 504, 508: Das Urteil Wasserleitungsverband Nördliches Burgenland präzisiert, wann eine Person unmittelbar betroffen und deshalb klagebefugt ist.

32) Dörr/Lenz, Europäischer Verwaltungsrechtsschutz, 2. Aufl. 2019, Rdnr. 536; Groß, Die Verwaltung 43 (2010), 349, 373; Calliess, NVwZ 2006, 1, 4. Insoweit kann der Auffassung von Köck/Henn, NVwZ 2020, 504, 509, wonach die Entscheidung über die Einordnung der Klagebefugnis eines Wasserversorgers in die Systematik des $₫ 42$ Abs. 2 VwGO dahinstehen könne, nicht gefolgt werden; wie gezeigt, zwingen schon die Maßstäbe des EuGH zur Differenzierung zwischen Verbands- und Individualklägern. Gleiches muss dann für die Integration in das nationale Recht gelten.

33) Unionsrechtlicher Hintergrund der Lärmaktionsplanung ist die Richtlinie 2002/49/EG des Europäischen Parlaments und des Rates vom 25.6.2002 über die Bewertung und Bekämpfung von Umgebungslärm, ABl. 2002 L 189, S. 12, zuletzt geändert durch die Richtlinie (EU) 2020/367 der Kommission vom 4.3.2020, AB1. 2020 L 67, S. 132

34) Vgl. hierzu und zum Vorstehenden BVerwG, Urt. v. 28.11.2019 - 7 C 2.18, juris Rdnr. 1 f., $7 \mathrm{f}$. 
tens könne eine Klagebefugnis nicht auf Art. 9 Abs. 3 AK als andere gesetzliche Bestimmung i.S.d. $\int 42$ Abs. 1 1. Hs VwGO gestützt werden, da diese Privilegierung Verbandsklägern vorbehalten sei. ${ }^{35}$ Zweitens komme ein nach $\ 42$ Abs. 2 2. Hs. VwGO zur Klage berechtigendes subjektives Recht nicht in Betracht, da die Pflicht zur Erstellung von Lärmaktionsplänen aus $₫ 47 \mathrm{~d}$ BImSchG ausschließlich Behörden adressiere und keine Rechte Dritter begründe. ${ }^{36}$ $\int 47 \mathrm{~d}$ BImSchG sei auch nicht unionsrechtskonform auszulegen, da die Umgebungslärmrichtlinie 2002/49/EG keine konkreten Grenzwerte fordert und deshalb nicht hinreichend bestimmt sei, um Rechte Einzelner zu vermitteln; hierin unterscheide sie sich wesentlich von der Luftreinhalteplanung oder den Pflichten aus der Nitrat-RL. ${ }^{37}$ Selbst jenseits der Frage nach der Bestimmtheit der Umgebungslärmrichtlinie sei - drittens - der Ermessensspielraum der planenden Behörden so weit, das Maß seiner Steuerung so gering, dass subjektive Rechte Dritter nicht entstehen könnten. ${ }^{38}$

Die Auffassung des BVerwG fügt sich in seine ständige Rechtsprechung ein, die die fehlende individualschützende Wirkung der Lärmaktionspläne im Kern mit dem Fehlen präziser und hinreichend klarer Grenzwerte begründet und so auf eine fehlende Quantifizierbarkeit der einzuklagenden Pflichten verweist. ${ }^{39}$ Teile der Literatur folgen diesem Ansatz. ${ }^{40}$ Soweit demgegenüber eine individualschützende Wirkung der Lärmaktionsplanung angenommen wird, wird deren gesundheitsschützende Zielsetzung zugrunde gelegt. Die Bedeutung der Lärmaktionsplanung erschöpfe sich nicht in behördlichen Planungspflichten, sondern solle nach dem Willen des Unionsgesetzgebers auch Einzelne vor den Auswirkungen von Lärm schützen. ${ }^{41}$ Es kommt hiernach weniger auf den Grad der Konkretisierung der Planungspflichten als vielmehr darauf an, dass ihre unionsrechtliche Grundlage auch dem Schutz des Einzelnen dient. Dies konnte angesichts des Wortlauts des Art. 3 lit. a), b) der RL 2002/49/EG, der gesundheitsschädliche Geräusche bzw. Auswirkungen ausdrücklich nennt, schon vor der Entscheidung des EuGH in der Rs. C-197/18 überzeugen. Mit dieser Entscheidung, die im Kontext des Wasserrechts ebenfalls auf die gesundheitsschützende Zielsetzung der Nitrat-RL abstellt, ${ }^{42}$ steht der Herleitung subjektiver Rechte in der Lärmaktionsplanung nun ein weiteres gewichtiges Argument zur Seite. Einiges spricht deshalb dafür, die Klagebefugnis Einzelner zu bejahen, ${ }^{43}$ insbesondere, wenn sie - wie im vom BVerwG beurteilten Fall - in einer Lärmschutzzone wohnen und so als unmittelbar betroffen i.S.d. Unionsrechts gelten. Damit ist noch nichts über die konkreten Maßnahmen, zu deren Ergreifung gerichtlich verpflichtet werden kann, gesagt; in diesem Zusammenhang sind dann die erheblichen behördlichen Ermessensspielräume bei der Planerstellung und Maßnahmenauswahl zu berücksichtigen. ${ }^{44}$ Dies kann indes nicht als Argument dienen, schon den Zugang zu Gericht zu verwehren.

\section{Schluss}

Die Entscheidung des EuGH in der Rs. Wasserleitungsverband Nördliches Burgenland dürfte den vorläufigen Schlusspunkt der Rechtsprechung zur Reichweite von Art. 9 Abs. 3 AK i.V.m. Art. 47 GRCh bilden. ${ }^{45}$ Sie legt die Maßstäbe für die Klagebefugnis Einzelner im europäisierten Umweltrecht fest. Sie lassen sich dergestalt zusammenfassen, dass Individualkläger zur Rüge objektiver Verletzungen des Unionsumweltrechts befugt sind, wenn sie unmittelbar betroffen sind. Diese Betroffenheit kann etwa in Gestalt eines aufgrund der Rechtsverletzung eingeschränkten Nutzungsrechts - rechtlicher oder - etwa in Gestalt einer konkreten Gesundheitsgefährdung - tatsächlicher Art sein. Das Gegenstück zur hier besprochenen Entscheidung ist die Protect-Entscheidung, die den
Umfang der Klagebefugnis von Verbänden auf Grundlage bestimmt. Dass beide Rechtsprechunglinien das deutsche Recht weiter unter Anpassungsdruck setzen, zeigt neben der Entscheidung des BVerwG zur Lärmaktionsplanung ein Vorlagebeschluss des VG Schleswig, der im Kontext einer Verbandsklage die Vereinbarkeit des $\$ 1$ Abs. 1 S. 1 Nr. 5 UmwRG mit dem Unions- und Völkerrecht zum Gegenstand hat. ${ }^{46}$ Mag der EuGH den unionsrechtlich gebotenen Umfang der Individual- und Verbandsklagerechte also vorerst abschließend definiert haben, ist ihre Ausgestaltung im deutschen Recht noch in der Entwicklung begriffen. ${ }^{47}$

Open Access. Dieser Artikel wird unter der Creative Commons Namensnennung 4.0 International Lizenz veröffentlicht, welche die Nutzung, Vervielfältigung, Bearbeitung, Verbreitung und Wiedergabe in jeglichem Medium und Format erlaubt, sofern Sie den/die ursprünglichen Autor(en) und die Quelle ordnungsgemäß nennen, einen Link zur Creative Commons Lizenz beifügen und angeben, ob Änderungen vorgenommen wurden.

Die in diesem Artikel enthaltenen Bilder und sonstiges Drittmaterial unterliegen ebenfalls der genannten Creative Commons Lizenz, sofern sich aus der Abbildungslegende nichts anderes ergibt. Sofern das betreffende Material nicht unter der genannten Creative Commons Lizenz steht und die betreffende Handlung nicht nach gesetzlichen Vorschriften erlaubt ist, ist für die oben aufgeführten Weiterverwendungen des Materials die Einwilligung des jeweiligen Rechteinhabers einzuholen.

Weitere Details zur Lizenz entnehmen Sie bitte der Lizenzinformation auf http://creativecommons.org/licenses/by/4.0/deed.de.

Open Access funding enabled and organized by Projekt DEAL.

35) BVerwG, Urt. v. 28.11.2019 - 7 C 2.18, juris Rdnr. $12 \mathrm{ff}$

36) BVerwG, Urt. v. 28.11.2019- 7 C 2.18, juris Rdnr. 17 m.w. N. aus der zugehörigen $\mathrm{R}$ spr.

37) BVerwG, Urt. v. 28.11.2019 - 7 C 2.18, juris Rdnr. 19 ff., insbes. 22. Näher zum Fehlen konkreter Grenzwerte in der Umgebungslärmrichtlinie Schulze-Fielitz/Wickel, in: Führ (Hrsg.), GKBImSchG, 2. Aufl. 2019, §47c Rdnr. 32 f.; Wysk, in: Giesberts/ Reinhardt (Hrsg.), BeckOK Umweltrecht, 55. Edition 2020, \$47c BImSchG Rdnr. 18ff.; Cancik, NVwZ 2008, 167, 169.

38) BVerwG, Urt. v. 28.11.2019 - 7 C 2.18, juris Rdnr. $23 \mathrm{f}$.

39) Z.B. BVerwG, Urt. v. 12.11.2014 - 4 C 34.13, NVwZ 2015, 596, Rdnr. 24; BVerwG, Beschl. v. 7.1.2019 - 7 B 16.18, juris Rdnr. 10; s. für einen Überblick über die (Instanz-)Rechtsprechung ferner Cancik, WiVerw 2012, 210, $219 \mathrm{ff}$.; Cancik, in: Landmann/Rohmer (Begr.), Umweltrecht, 71. EL 2014, \47d BImSchG Rdnr. $32 \mathrm{ff}$.

40) Z.B. Schulze-Fielitz/Wickel, in: Führ (Hrsg.), GK-BImSchG, 2. Aufl. 2019, \47d Rdnr. 94 ff.; Jarass, BImSchG, 13. Aufl. 2020, $\$ 47 \mathrm{~d}$ Rdnr. 21; zurückhaltend in Bezug auf die Herleitung von Ansprüchen Einzelner auch Blaschke, Lärmminderungsplanung, 2010, S. 389 ff., insbes. S. 394; Kröner, UPR 2013, 89 ff.; nunmehr auch Wysk, in: Giesberts/Reinhardt (Hrsg.), BeckOK Umweltrecht, 55. Edition 2020, $\$ 47 \mathrm{~d}$ BImSchG Rdnr. $33 \mathrm{f}$.

41) Cancik, ZUR 2007, 169, 172 ff.; Schlacke, NVwZ 2015, 563, 565; Sparwasser/Engel, NVwZ 2010, 1513, 1518f.; s.a. Saip, Lärmschutz durch Lärmaktionsplanung, 2019, S. $246 \mathrm{ff}$.

42) EuGH, Urt. v. 3.10.2019 - C-197/18, NuR 2019, 751 (Wasserleitungsverband Nördliches Burgenland), Rdnr. $36 \mathrm{f}$.

43) In diese Richtung auch Berkemann, DVB1. 2020 (i.E.); a.A Kment, NVwZ 2020, 894, $895 \mathrm{f}$

44) Cancik, ZUR 2007, 169, 173 f.; s.a. Sparwasser/Engel, NVwZ 2010, 1513, 1519, die deshalb einen Anspruch auf Aufnahme konkreter Maßnahmen für ausgeschlossen halten. Allg. näher zum Planungsermessen i. R.d. Lärmaktionsplanung Saip, Lärmschutz durch Lärmaktionsplanung, 2019, S. $143 \mathrm{ff}$.

45) Dies lässt sich auch daran ablesen, dass der EuGH sie in einer neueren Entscheidung zum Wasserrecht ohne Modifikationen zugrunde gelegt hat, s. EuGH, Urt. v. 28.5.2020 - C-535/18, ECLI:EU:C:2020:391 - IL u.a. gegen Land Nordrhein-Westfalen, Rdnr. $120 \mathrm{ff}$

46) VG Schleswig, Beschl. v. 20.11.2019 - 3 A 13/18, ZUR 2020, 106.

47) So auch Berkemann, DVB1. 2020 (i.E.). 